\title{
Impact of a nurse navigator on a dedicated inflammatory bowel disease-focused gastroenterology clinic
}

\author{
Parth Maheshwaria, Anne Bobbb, August Stuartc, Shannon Dalessio', Nana Bernaskoc, Andrew Tinsleyc, \\ Kofi Clarked, Matthew D. Coatesc*, Emmanuelle Williams**
}

Pennsylvania State University College of Medicine, Hershey, PA, USA

\begin{abstract}
Background Management of inflammatory bowel disease (IBD) patients can be very challenging. Nurse navigators (NNs) have demonstrated great promise for coordinating care of complex disorders but little is known about their impact on the setting of IBD. We undertook this study to evaluate how the introduction of a NN in a dedicated IBD clinic would influence several outcomes related to patient care.
\end{abstract}

Methods A retrospective chart review was performed evaluating Penn State IBD clinic patients receiving care a year before and after the introduction of a dedicated NN to the clinic. No-show rates, new appointments in less than 21 days, total clinic visits per month, and patient enrolment in our IBD registry and biorepository were measured prior to and after hiring of the NN between 2 providers. Each provider and their composite data were statistically compared using univariate analysis.

Results After hiring the NN, there was a statistically significant decrease in combined no-show rates $(\mathrm{P}=0.02)$. There was no significant difference in the combined average number of new appointments in less than 21 days $(\mathrm{P}=0.62)$ or total clinic visits per month $(\mathrm{P}=0.09)$. Enrolment in the database and biorepository increased (from $83 \%$ to $90 \%$ ). Finally, $97 \%$ were satisfied with the NN's services, and $94 \%$ were satisfied with the IBD education they provided.

Conclusions Hiring a NN in our clinic was associated with high patient satisfaction, reduced noshow rates, and increased research participation. Thus, incorporation of a NN can improve care in an IBD-centered gastroenterology clinic.

Keywords Inflammatory bowel disease, nurse navigator, Crohn's disease, ulcerative colitis, noshow rates

Ann Gastroenterol 2021; $34(x): 1-5$

\section{Introduction}

Inflammatory bowel disease (IBD) includes a set of chronic inflammatory disorders of the gastrointestinal tract, including

Department of ${ }^{a}$ Medicine (Parth Maheshwari); ${ }^{\text {SSurgery, Division of }}$ Colorectal Surgery (Anne Bobb); ${ }^{\mathrm{c} M e d i c i n e, ~ D i v i s i o n ~ o f ~ G a s t r o e n t e r o l o g y ~}$ and Hepatology (August Stuart, Shannon Dalessio, Nana Bernasko, Andrew Tinsley, Kofi Clarke, Matthew D. Coates, Emmanuelle Williams), Pennsylvania State University College of Medicine, Hershey, PA, USA

${ }^{\star}$ These authors contributed equally to this manuscript

Conflict of Interest: None

Correspondence to: Emmanuelle Williams, MD, 500 University Drive, Hershey, PA 17033, e-mail: ewilliams3@pennstatehealth.psu.edu

Received 28 October 2020; accepted 27 November 2020; published online 27 May 2021

DOI: https://doi.org/10.20524/aog.2021.0627
Crohn's disease (CD) and ulcerative colitis (UC). In recent years, many advancements have been made in the quantity and quality of diagnostic and therapeutic options available to individuals suffering from these disorders. Unfortunately, the underlying pathophysiology of IBD remains unclear and its management can be challenging. Additionally, even when the gastrointestinal manifestations of these disorders are successfully controlled, a variety of extraintestinal manifestations (arthropathies, dermatopathies, and psychiatric conditions) can still cause morbidity [1]. Thus, to keep individuals with IBD as healthy as possible, it is necessary to consistently monitor their progress, adjust their medical regimen as needed, and coordinate their often complex care with other specialists. Optimized care is thus often difficult, even for the relatively healthy patient.

IBD providers use a variety of methods to address these challenges. For example, many providers have given patients open access to their electronic medical records system to allow for more flexibility in scheduling, while others have established 
IBD "homes" to provide comprehensive care [2]. However, one approach that has not been widely adopted or studied in IBD centers is the use of a nurse navigator (NN). Generally speaking, the NN's duty is to help coordinate patients' ongoing treatment and follow up of disease management. This includes appointment scheduling/reminding, helping patients with insurance paperwork, providing various options for support (dietitians, psychiatrists), compliance assessment, and medication and disease education [3].

Studies involving NNs have been most frequently undertaken in the setting of oncology centers. For example, a 2010 Swedish study demonstrated that incorporation of a $\mathrm{NN}$ in a breast cancer outpatient care center led to reduction in wait times and visit lengths along with significant improvements in patient satisfaction [4]. Another study performed at Mayo Clinic in Jacksonville, FL, showed that the introduction of a $\mathrm{NN}$ in their breast mammography suite was associated with quicker identification of benign lesions (usually the same day) [5]. There are also reports of radiology departments using NNs to assist with clinical workflow. Radiology nurses in Edmonton, Alberta, help coordinate appointments for patients who live in rural provinces of the Yukon, Northwestern Territories, and Nunavut to ensure they attend their appointments and receive their results [6].

At our IBD center, we incorporated a NN who ensures patients are fully chaperoned throughout their medical care from their initial referral to their ongoing care. This includes coordinating appointments in our combined gastrointestinal and colorectal surgery clinics for drug initiations, infusions, post-treatment follow up, streamlining referrals to other specialties, providing patient education and clinical trial enrollment. The purpose of this study was to examine the impact of a NN on multiple key clinical outcomes in our center. Based upon the findings of previous studies, we expected to see a positive impact on several clinical metrics, including patient no-show rates, new patient appointments, and overall patient satisfaction in their care.

\section{Patients and methods}

\section{Study population}

We performed a retrospective cohort study using information from the electronic health record of our institution's IBD Center from 2014-2015. Our institution is a tertiary care center that cares for at over 6000 patients with a diagnosis of IBD. This study was performed with the oversight and permission of the local Institutional Review Board.

\section{Definitions and data}

In 2014, a single NN was hired by the our IBD Center to help triage phone calls, contact new patients, coordinate services (specialty referrals, appointments, infusions, studies, consults), educate patients, provide support as well as referral to our support group, enroll individuals in clinical trials, and assist in transition of patients from the pediatric IBD clinic to the adult IBD clinic. Patients included in the study had an established diagnosis of IBD (CD, UC, or IBD colitis) based on standard clinical criteria.

Several outcomes were compared between cohorts of patients seen by 2 gastroenterology providers in our IBD center prior to and after the incorporation of a NN including: (a) noshow rates per month; (b) new appointment wait times for the first visit (recorded as more or less than 21 days); (c) total clinic visits per month; and (d) rate of patient enrolment in a dedicated IBD database and biorepository. Additionally, a postvisit survey was done to assess patient satisfaction with the NN.

\section{Statistical analysis}

Data was extracted and analyzed using Graphpad Prism (version 7). No-show rates, new appointment wait times, total clinic visits per month, and IBD database and biorepository recruitment rates were each separately compared between cohorts seen prior to and after hiring a $\mathrm{NN}$ using an unpaired Student's $t$-test or Chi-square test as appropriate. The data associated with 2 different providers were evaluated separately and together in this study as possible and appropriate. Values listed represent means \pm standard deviation (SD) unless indicated otherwise. Statistical significance was defined as a $\mathrm{P}$-value of less than 0.05 .

\section{Results}

\section{Demographics and clinical characteristics}

570 patients cared for at our IBD center were included in this study. 170 patients $(29.8 \%)$ were managed by our providers before the hiring of the NN. Of these individuals, 76 were male (44.7\%), and 94 were female (55.3\%). 96 patients had CD (56.5\%), 66 patients had UC (38.8\%), and 8 patients had indeterminate colitis ([IC] 4.7\%). The average age of these patients was 39.8 years (Table 1). 400 study participants were managed by our providers after the NN was hired. Of these patients, 159 were male (39.8\%), and 241 were female (60.2\%). 216 patients had CD (54.0\%), 151 patients had UC (37.8\%), and 33 patients had IC (8.2\%). The average age of these patients was 38.7 years (Table 1 ).

\section{No-show rates per month}

Prior to hiring the NN, provider 1 had an average no-show rate of $16.5 \pm 7.1 \%$ per month. After hiring the NN, their noshow rate dropped to $8.1 \pm 3.2 \%$ per month $(\mathrm{P}=0.004)$. Provider 2 had a no-show rate of $10.6 \pm 4.1 \%$ per month prior to hiring 
Table 1 Demographic and clinical characteristics of nurse navigator cohort

\begin{tabular}{lcccc}
\hline Characteristics & $\begin{array}{c}\mathrm{n} \text { or } \\
\text { mean } \\
\mathrm{n} \pm \mathrm{SD}\end{array}$ & $\begin{array}{c}\text { Prior to } \\
\text { nurse } \\
\text { navigator }\end{array}$ & $\begin{array}{c}\text { After } \\
\text { nurse } \\
\text { navigator }\end{array}$ & P-value \\
\hline Sample (F/M) & $\begin{array}{c}570 \\
(335 / 235)\end{array}$ & $\begin{array}{c}170 \\
(94 / 76)\end{array}$ & $\begin{array}{c}400 \\
(241 / 159)\end{array}$ & 0.31 \\
& $312 / 217 / 41$ & $96 / 66 / 8$ & $216 / 151 / 33$ & 0.32 \\
$\begin{array}{l}\text { Disease type } \\
\text { (CD/UC/IC) }\end{array}$ & $39.0 \pm 14.2$ & $39.8 \pm 14.0$ & $38.7 \pm 14.1$ & 0.43 \\
\hline $\begin{array}{l}\text { Age } \\
\text { (years } \pm \mathrm{SD})\end{array}$ & & & & \\
\hline
\end{tabular}

F/M, female/male; $C D$, Crohn's disease; UC, ulcerative colitis; IC, indeterminate colitis; $S D$, standard deviation

the $\mathrm{NN}$, and an average no-show rate of $10.4 \pm 6.0 \%$ after hiring $(\mathrm{P}=0.943)$. When compared to provider 2, there was a trend toward a greater no-show rate for provider 1 prior to hiring the $\mathrm{NN}(\mathrm{P}=0.060)$ but no significant difference in this rate after hiring the $\mathrm{NN}(\mathrm{P}=0.290)$. When considered together, the providers had a mean no-show rate of $27.1 \pm 7.6 \%$ prior to hiring the $\mathrm{NN}$, and mean of $18.5 \pm 6.9 \%$ after hiring $(\mathrm{P}=0.020)$ (Fig. 1).

\section{New appointment wait times}

Provider 1 had an average of 3.6 \pm 1.3 new appointments per month with lead times less than 21 days prior to hiring the NN. After hiring, this increased to 9.0 \pm 4.1 new appointments per month $(\mathrm{P}=0.002)$. Provider 2 had an average of $6.6 \pm 3.5$ new appointments per month with lead times less than 21 days prior to the $\mathrm{NN}$, and an average of $4.0 \pm 3.3$ new appointments per month after hiring $(\mathrm{P}=0.252)$. Provider 2 had a larger number of appointments with lead times less than 21 days compared to Provider 1 before hiring a NN $(\mathrm{P}=0.040)$. There was not a significant difference in this parameter between the 2 providers after hiring the $\mathrm{NN}(\mathrm{P}=0.120)$. Together, both providers had an average of $10.2 \pm 3.4$ new appointments per month prior to the $\mathrm{NN}$, and an average of $13.0 \pm 6.2$ new appointments per month after the NN ( $\mathrm{P}=0.621)$ (Fig. 2).

\section{Total clinic visits per month}

The average number of clinic visits per month for provider 1 increased from $54.0 \pm 19.2$ visits per month to $75.0 \pm 14.6$ visits per month after hiring the $\mathrm{NN}(\mathrm{P}=0.030)$. Provider 2 had $43.0 \pm 10.8$ visits per month prior to the $\mathrm{NN}$, and $44.0 \pm 15.9$ visits after hiring the $\mathrm{NN}(\mathrm{P}=0.572)$. Each provider had statistically similar clinic visits per month before hiring a NN $(\mathrm{P}=0.160)$, but Provider 1 had a significantly higher number of clinic visits after the hiring $(\mathrm{P}=0.002)$. Together, both providers did not see a significant increase in total clinic visits per month, with an average of $97.0 \pm 25.0$ visits per month prior to hiring, and $119.0 \pm 23.9$ visits per month after hiring $(\mathrm{P}=0.090)$ (Fig. 3).

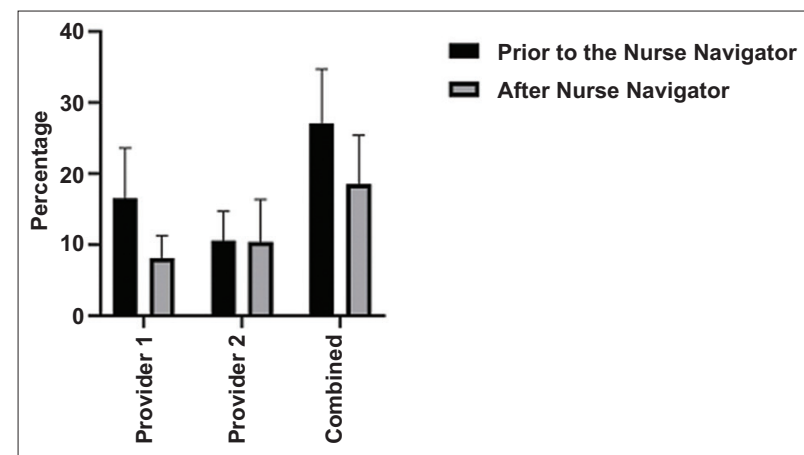

Figure 1 No-show rate per month. Together providers 1 and 2 had a significant decrease in no-show rates after hiring the nurse navigator

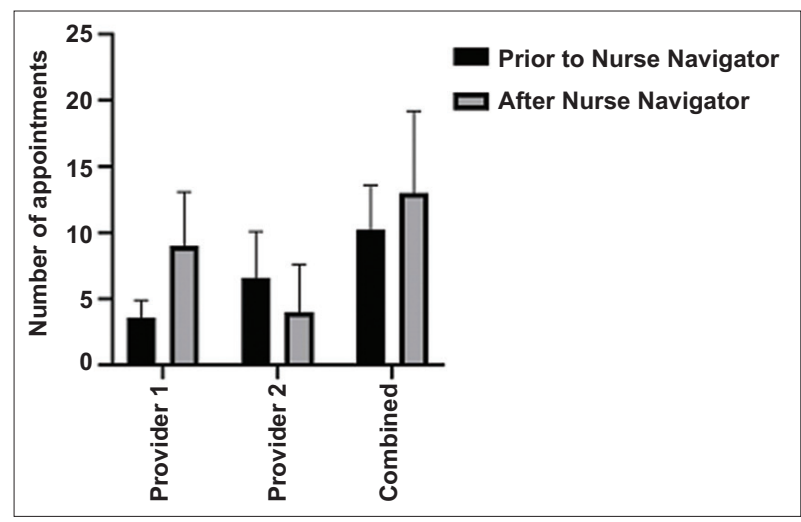

Figure $2 \mathrm{New}$ patient visits. While provider 1 had a significant increase in new clinic visits per month with a lead time of 21 days, provider 2 and the combination of both providers did not have a significant increase prior to and after hiring the nurse navigator

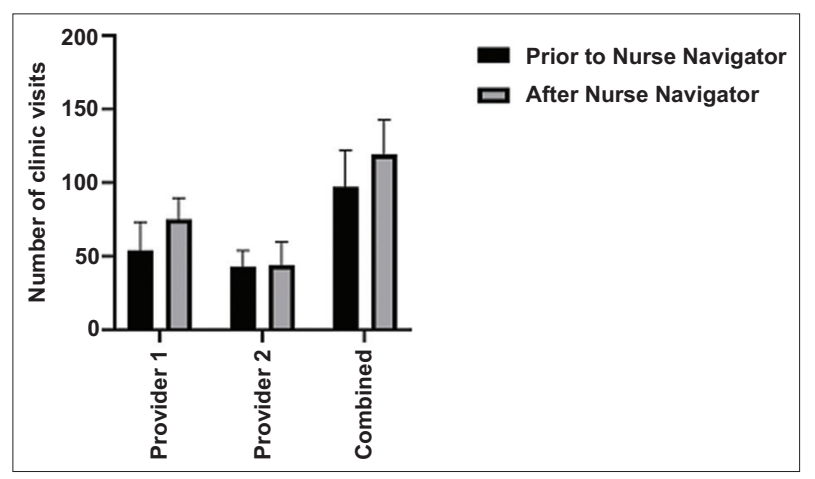

Figure 3 Total clinic visits per month. While provider one had a significant increase in total clinic visits per month, there was no significant increase with provider 2 or the combination of both providers prior to and after hiring the nurse navigator

\section{IBD database and biorepository recruitment}

$141(82.9 \%)$ of the patients evaluated before incorporation of a NN were enrolled in the IBD database and biorepository. After the incorporation of the $\mathrm{NN}$, a significantly higher proportion [ $\mathrm{n}=360(90.0 \%) ; \mathrm{P}=0.02]$ of patients were enrolled (Fig. 4). 


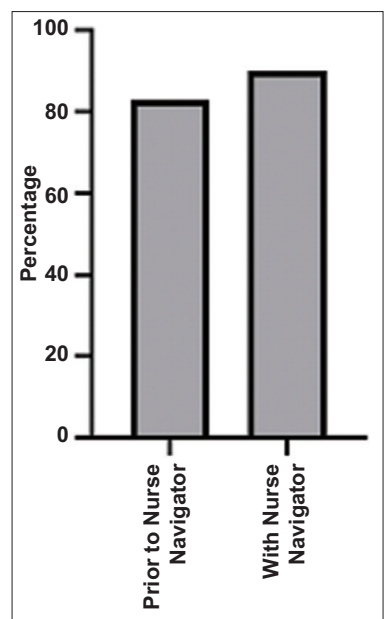

Figure 4 Inflammatory bowel disease data registry and bio-bank enrolment. Increased from $83 \%$ (before introduction of the nurse navigator) to $90 \%$ (after introduction of the nurse navigator)

\section{Patient satisfaction}

Of note, $97 \%$ of all patients included in this study were satisfied with the NN's services. Additionally, 94\% of patients were satisfied with the education regarding IBD that the NN provided.

\section{Discussion}

This is one of the first studies to examine the effects of a NN in a dedicated IBD clinic. Importantly, we demonstrated that the incorporation of a $\mathrm{NN}$ in our clinic was associated with reduced patient no-show rates and increased recruitment to our patient IBD database and biorepository. Patients also reported high satisfaction rates with their care in the setting of a NN.

The reduction in no-show rates in the IBD clinic is important, as there are many associated benefits. First, the patient receives better care as a result of having more consistent access to their providers. Second, increased revenue is generated for the medical facility and institution. Lastly, attendance at scheduled appointments results in an improved relationship between the patient and physician [7], allowing more time to explain the benefits of various treatment options and follow-up visits. This also leads to a decrease in frustration for physicians, as physicians can only provide optimal care to patients when scheduled appointments are maintained [8].

In the case of our clinic, we believe no-show rates decreased for several reasons. First, our NN prioritized patients that required urgent or emergent care. Second, she coordinated colorectal surgery and gastroenterology visits when appropriate so that medical and surgical interventions could be expedited and done simultaneously when required. Third, patients were screened for risk factors related to no shows (including assessing challenging familial situations, transport issues, etc.), facilitating targeted selection of appointments to adjust to patient needs.
It is also notable that the use of a $\mathrm{NN}$ also increased recruitment in our research database and biorepository. This resource has been instrumental in growing our program's research efforts and helped to provide us a platform upon which to coordinate research with other IBD investigators (e.g., Crohn's and Colitis Foundation IBD Qorus program). This work also allows our team to more efficiently address quality initiatives, which aid in improving care for our patients [9].

Inclusion of a NN may also have a potential financial benefit, as it could reduce health care costs often associated with hospitalizations and complications (e.g., surgery, nosocomial infections, etc.). Notably, NNs have been extensively utilized in oncology centers without demonstrating a significant increase in charges or reimbursements. This appears to be the case with IBD as well. One study showed that there was no significant increase in reimbursements and charges [10]. This has been evident even when considering care of patients with relatively lower incomes. Researchers at the University of Pittsburgh Medical Center in Pittsburgh, PA showed that, in low-income populations, a NN allowed the practice to assume fewer costs as the navigator helped in patient education and outpatient follow-up coordination [11].

We recognize several limitations in this study. We were not able to control for some important clinical disease characteristics, such as disease severity. For example, lead times may have also been affected by disease severity. The NN often prioritized follow-up appointments for patients admitted to the hospital for IBD flares and subsequently required close monitoring as an outpatient. By prioritizing these patients, disease severity may have had a confounding effect on lead times. We were also only able to parse out data for 2 IBD providers associated with this center. As described above, some of the study outcomes were different between the 2 providers. For example, despite the lack of a significant change in mean new appointment wait times and clinic visits per month, these variables demonstrated statistically significant differences in association with one of the providers. It is possible that differences between the providers in overall clinic volume, subspecialty focus, time at the clinic, and/or even patient rapport drove these variations. It is also possible that the $\mathrm{NN}$ put more effort into one provider's clinic operation during the study period compared to the other. However, it is difficult to make definitive determinations in this regard given the nature of the data being evaluated and the small sample size. It would be helpful to evaluate data for other providers to see if similar differences were found and whether particular elements of care impacted the likelihood of improvement in these metrics. Additionally, we only followed patients for a relatively short time period ( $<1$ year). It would be helpful to know if these improvements have persisted. Finally, it would be critical in future studies to measure the impact the NN has on IBD activity, complications, and healthcare resource utilization.

Despite these shortcomings, this study demonstrated that the incorporation of a NN in our IBD clinic has been associated with multiple beneficial clinical outcomes. Although there is certainly much more to study in this context, it is already clear to us that our $\mathrm{NN}$ has made a significantly positive impact on the quality of patient care in a variety of ways. Particularly 
when considering this era of increasingly patient-centered and cost-conscious care, our findings and experience strongly support the incorporation of a NN in IBD clinic settings.

\section{Summary Box}

\section{What is already known:}

- Successful management of inflammatory bowel disease (IBD) relies on the expert care of multiple providers, often from a variety of specialties

- Nurse navigators (NNs) have been utilized in oncology clinics to help patients navigate complex care plans

- There has not been any full investigation into the utility of a NN in an IBD clinic

\section{What the new findings are:}

- Introduction of a NN in our dedicated IBD clinic was associated with significantly decreased noshow rates for appointments

- While not statistically significant, after introduction of a NN, there was an increase in new patient appointments, with lead times less than 21 days, and in the total number of clinic visits per month

- Patients had a high rate of satisfaction when a NN was involved in their care

\section{References}

1. Eichele DD, Young R. Medical management of inflammatory bowel disease. Surg Clin North Am 2019;99:1223-1235.

2. Regueiro M, Click B, Holder D, Shrank W, McAnallen S, Szigethy E. Constructing an inflammatory bowel disease patient-centered medical home. Clin Gastroenterol Hepatol 2017;15:1148-1153.

3. Pautasso FF, Zelmanowicz AM, Flores CD, Caregnato RCA. Role of the nurse navigator: integrative review. Rev Gaucha Enferm 2018;39:e20170102.

4. Bergenmar M, Nylén U, Lidbrink E, Bergh J, Brandberg Y. Improvements in patient satisfaction at an outpatient clinic for patients with breast cancer. Acta Oncol 2006;45:550-558.

5. Palmieri FM, DePeri ER, Mincey BA, et al. Comprehensive diagnostic program for medically underserved women with abnormal breast screening evaluations in an urban population. Mayo Clin Proc 2009;84:317-322.

6. Brown $\mathrm{CD}$. Improving patient care in the diagnostic imaging department: the role of the nurse navigator. J Radiol Nurs 2012;31:97-100.

7. Gariti P, Greenstein RA, Olsen K, Harris PE. Scheduled appointments and patient-staff compliance. Am J Orthopsychiatry 1987;57:135-137.

8. Pini A, Sarafis P, Malliarou M, et al. Assessment of patient satisfaction of the quality of health care provided by outpatient services of an oncology hospital. Glob J Health Sci 2014;6:196-203.

9. Kearney A, Harman NL, Rosala-Hallas A, et al. Development of an online resource for recruitment research in clinical trials to organise and map current literature. Clin Trials 2018;15:533-542.

10. Ritz LJ, Nissen MJ, Swenson KK, et al. Effects of advanced nursing care on quality of life and cost outcomes of women diagnosed with breast cancer. Oncol Nurs Forum 2000;27:923-932.

11. Schwaderer KA, Itano JK. Bridging the healthcare divide with patient navigation: development of a research program to address disparities. Clin J Oncol Nurs 2007;11:633-639. 